\title{
Uso de la Plataforma Classroom como herramienta virtual a nivel de Educación media general
}

\author{
Use of the Classroom Platform as a virtual tool at the level of General Middle \\ Education
}

\author{
a Ángel Yosmar Polo Hurtado \\ poloangel9@gmail.com \\ Código ORCID: 0000-0002-4311-9461
}

\author{
a María de los Ángeles Gallardo Sánchez \\ m.delosangeles.g@hotmail.com \\ Código ORCID: 0000-0001-8912-3274
}

\section{Secretaría de Educación y Deporte del Estado Carabobo, Venezuela}

\author{
Artículo recibido en febrero 2020 | Arbitrado en marzo 2020 | Publicado en mayo 2020
}

\section{Resumen}

La finalidad de esta investigación fue proponer el uso de una herramienta virtual denominada Classroom, para la implementación efectiva del proceso de enseñanza-aprendizaje en la asignatura de Informática en los estudiantes de tercer año de la E.T.R. "Monseñor Gregorio Adam". Esta propuesta se fundamentó en las teorías del constructivismo de Piaget (1970) y Teoría de la Cibernética (1948). Fue un estudio descriptivo, de campo. La población y muestra estuvo representada por treinta estudiantes, empleándose la técnica de la encuesta y como instrumento se aplicó un cuestionario de respuestas dicotómicas. La confiabilidad se determinó por el coeficiente Kuder Richardson, quedando en 0,61. El análisis de datos se procesó a través de interpretaciones estadísticas porcentuales, arrojando la necesidad de implementar la herramienta virtual Classroom para facilitar la productividad del aprendizaje de la asignatura. Concluyendo, esta plataforma servirá de apoyo y guía para hacer el proceso educativo efectivo en los contenidos

\footnotetext{
Abstract

Virtual Tool, called Classroom, for the effective implementation of the teachinglearning process in the subject of Computer Science in third-year students of the E.T.R. "Monsignor Gregorio Adam". This proposal was based on Piaget's (1970) and Cybernetics (1948) theories of constructivism. It was a descriptive, field study. The population and sample were represented by thirty students, using the survey technique and a dichotomous response questionnaire was applied as an instrument. Reliability was determined by the Kuder Richardson coefficient, reaching 0.61 . The data analysis was processed through percentage statistical interpretations, showing the need to implement the virtual Classroom tool to facilitate the productivity of learning the subject. In conclusion, this platform will serve as support and guide, to make the educational process effective in the programmatic contents.
}

\section{Palabras clave:}

Tecnología; educación; herramientas; enseñanza; aprendizaje

\section{Keywords:}

Technology;

Education; Tools; Teaching; Learning 


\section{INTRODUCCIÓN}

Con el paso del tiempo las herramientas virtuales utilizadas en la educación han ido evolucionando y actualizándose paulatinamente, considerando para ello los avances tecnológicos que se han manifestado en todo el mundo. Así pues, se evidencia en la actualidad el incremento y el uso de las herramientas virtuales que permiten ampliar el proceso de enseñanza-aprendizaje que anteriormente era limitado por un intervalo de tiempo y espacio físico específico. Los nuevos instrumentos virtuales de la llamada "Educación 2.0" crean un sin fin de posibilidades para que el estudiante virtual acceda en cualquier momento y desde cualquier computador con acceso a la red o dispositivo de telefonía inteligente.

No obstante, las facilidades y ventajas que ofrecen las herramientas virtuales, al igual que en la educación presencial, requerirán para su implementación, por parte del estudiante, la aceptación y su compromiso en el proceso de autorregulación en la construcción de conocimientos. Por parte del docente implica actualizarse día a día en el uso correcto de la innumerable gama de instrumentos de la educación virtual.

En este sentido, la Organización de las Naciones Unidas para la Ciencia y Cultura (Unesco, 2008) explica que en los estándares de competencias TIC, "los docentes en ejercicio necesitan estar preparados para ofrecer a los estudiantes oportunidades de aprendizaje apoyadas en las TIC, para utilizarlas, de tal manera que éste pueda contribuir al aprendizaje de los estudiantes" (p. 2). Lo que implica que la educación es un proceso complejo como la cultura, porque ambas son resultado de la construcción social, formando la base para la concreción de transformaciones significativas del estudiantado.
Adicionalmente, la mencionada organización sostiene que el uso de las tecnologías podría permitir el mejoramiento en la productividad del aprendizaje, la misma se puede utilizar para la adquisición, producción, administración y difusión de información relacionada con el contenido académico, ya que está diseñado para apoyar las materias. El gran auge que ha tenido la tecnología de avanzada como lo es el uso del computador, obligando a pensar en la necesidad de analizar, por un lado, los efectos tecnológicos. Hoy en día son de gran importancia las herramientas virtuales para la educación, porque estas permiten romper paradigmas y a su vez implementar herramientas para hacer el proceso de enseñanza-aprendizaje dinámico y factible. Sin embargo, para la sociedad es de suma importancia porque les posibilita, entre diversos aspecto, conectarse, visitar y aprender nuevas culturas.

En Venezuela, siguiendo las directrices emanadas de la Unesco en el año 1999 con la promulgación de la Constitución de la República Bolivariana de Venezuela se materializa el deber de la inclusión de las tecnologías, al igual que la Ley Orgánica de Ciencia y Tecnología (2005), por consiguiente en el Artículo 1.

Dicha Ley nos refiere que en Venezuela la Educación y la Tecnología van de la mano con el proceso de enseñanza-aprendizaje, ya que estos permiten la formación del ciudadano para que puedan desenvolverse en su campo laboral y social, sin embargo el docente debe estar capacitado y actualizado a los nuevos cambios tecnológicos que se han venido dando durante estos últimos tiempos, ya que son muy importantes para la evolución del proceso educativo.

La tecnología emplea nuevas estrategias de aprendizaje, que les permite al docente de 
aula modificar el esquema tradicional que se ha venido llevando durante muchos años, para así transformarlo en uno más factible, sencillo y eficaz que responda a las necesidades de cada estudiante y poder formar individuos capaces de desenvolverse en cualquier campo de la vida, libres, autónomos y con valores, sirviendo de ejemplo para otras generaciones.

En el caso de la Escuela Técnica Robinsoniana "Monseñor Gregorio Adam", ubicada en el Municipio Naguanagua del Estado Carabobo, específicamente en el sector Barrio Unión, Calle Ruiz Pineda, cruce con 24 de Julio. № 104-28, fundado en el año 1970. En tercer "3er" año Sección "A" de Educación Técnica existe una matrícula de treinta (30) estudiantes, donde se imparte la asignatura: Informática, en la cual se pudo evidenciar, mediante un diagnóstico basado en la observación, las carencias que tienen los estudiantes para desarrollar actividades en la asignatura antes mencionada, ya que no poseen las habilidades, conocimientos y el uso adecuado de las herramientas virtuales que se pueden utilizar como medio para facilitar el proceso de enseñanza-aprendizaje de manera más efectiva, flexible y sencilla.

Por lo tanto, la falta del dominio de contenido y la incorporación de los recursos didácticos por parte del docente no hacen que despierte el interés del estudiantado, limitando así el desarrollo de las actividades propuestas, motivo por el cual se recomienda incorporar el uso de esta herramienta por ser la más adecuada en sus actividades académicas. A través de la aplicación de Google Classroom los docentes pueden crear clases, repartir deberes, enviar comentarios y tener acceso a todo desde un solo lugar, aumentando así, el interés y la atención de los estudiantes de tercer (3er) año Sección "A" de Educación Técnica, facilitando con ello el proceso de enseñanza-aprendizaje de manera significativa.

Debido a las observaciones efectuadas en el campo objeto del presente estudio, se evidenció que la institución educativa en referencia, a pesar de contar con la infraestructura y recursos necesarios, materiales y equipos, no hacen uso adecuado de ellas, dado que académica y vivencialmente adolecen de la formación requerida para implementar herramientas cónsonas con el uso de la pre descrita plataforma virtual, para completar con éxito el proceso enseñanza-aprendizaje.

En tal sentido, el objetivo general de esta investigación fue proponer el uso de la Plataforma Classroom como herramienta virtual en la asignatura de Informática en los estudiantes de 3er año "A" del E.T.R. "Monseñor Gregorio Adam". Los objetivos específicos fueron: Diagnosticar el nivel de conocimientos que poseen los estudiantes en el uso de las herramientas virtuales en el E.T.R. "Monseñor Gregorio Adam"; determinar la factibilidad de la implementación de la plataforma Classroom como herramienta virtual en la asignatura de Informática del E.T.R. "Monseñor Gregorio Adam", para llegar a diseñar un sistema de trabajo para la aplicación del Classroom como herramientas virtual en la asignatura de Informática del E.T.R. "Monseñor Gregorio Adam”.

Con la utilización de esta herramienta virtual en el ámbito educativo se fortalece el proceso de enseñanza-aprendizaje, como elemento auxiliar de la enseñanza, es una práctica generalizada en la sociedad moderna, motivado por su propio desarrollo tecnológico, entre otros factores. En tal sentido, las nuevas TIC han tenido un impacto en todas las áreas institucionales de la sociedad y en la educación media y técnica. En resumen, lo primordial de este estudio fue 
incorporar el uso de las TIC en el área de informática aplicando la herramienta virtual Classroom, para el desarrollo cognitivo de los estudiantes.

Debe señalarse que esta propuesta buscó que la institución educativa seleccionada se desarrolle y actualice en los conocimientos de "enseñar a aprender" capacidades, habilidades y destrezas que pueden ser adquiridos mediante la plataforma Classroom, ya que brinda una serie de opciones factibles para desarrollar las actividades académicas propuestas en el contenido programático respectivo. En este sentido beneficia a los estudiantes, pues ofrece las técnicas necesarias para realizar las actividades. Además, Classroom es el aula virtual que Google ha diseñado para completar las Google Apps para educación, con el objetivo de organizar y mejorar la comunicación entre docentes y estudiantes. Para el docente tiene gran utilidad, ya que puede lograr un mejor rendimiento y a su vez permite que el estudiantado se interese cada vez por actualizarse con estas herramientas.

A nivel social esta herramienta virtual es importante porque actualmente la sociedad vive en un cambio constante y por esto se debe romper los paradigmas tradicionales y generar un cambio a la sociedad utilizando las Tecnologías de Información y Comunicación (TIC), como herramientas en el proceso de enseñanza-aprendizaje de la vida diaria de cada estudiante. Por tanto, esta investigación servirá a futuras investigaciones, ya que su aporte es novedoso en las nuevas actualizaciones tecnológicas como recurso didáctico en el proceso educativo.

En relación al desarrollo de del presente estudio basado en las TIC en la E.T.R "Monseñor Gregorio Adam", se recopiló la incorporación de la herramienta virtual Classroom, en el ámbito educativo, y a su vez cómo podría crear una posibilidad de ampliar los medios de estrategias evaluativas en el proceso de enseñanza-aprendizaje para optimizar y alcanzar los objetivos propuestos por el docente. Para lograr este cometido se procedió, primeramente, a indagar los estudios que antecedieron al mismo, con el fin de fundamentarlo y ampliar la problematización.

\section{Estudios previos}

Se inició con el estudio de Sánchez (2017), titulado "Flipped Classroom. La clase invertida, una realidad en la Facultad de Ciencias de la Educación de la Universidad de Málaga", la cual se trató de una investigación de campo con nivel evaluativo.

La población objeto de estudio es el alumnado de la Facultad de Ciencias de la Educación de la Universidad de Málaga. En este caso se ha optado por un muestreo incidental o casual, seleccionando los individuos a los que se tiene fácil acceso. La muestra pertenece a las asignaturas de Didáctica de la Medida de los grupos A y B de $4^{\text {o }}$ curso y alumnos de ler curso de Tecnologías de la Comunicación y la Información Aplicadas a la Educación de Grado de Maestro de Educación Primaria, de los grupos B, C, D y F.

En el muestreo incidental se seleccionan de forma directa e intencionada los individuos de la población. El caso más frecuente de este procedimiento es tomar como muestra los propios estudiantes de los que disponen los profesores participantes o que se prestan a participar en la investigación, como ha sido el caso; la cual se le aplicó como técnica la encuesta y como instrumento un cuestionario contentivo de quince (15) preguntas abiertas y cerradas, que arrojaron como conclusión que los docentes no estaban aplicando estrategias 
virtuales más adecuadas para la enseñanzaaprendizaje del estudiante.

Por esto resulta necesario resaltar la relación que tiene con la presente investigación, ya que su objetivo es buscar el grado de satisfacción que tienen los estudiantes en el uso de la metodología Classroom en distintas disciplinas del aprendizaje y a su vez facilitando con su implementación la evaluación de los procesos de enseñanza-aprendizaje.

Por otra parte, en Venezuela, Agudo (2016), en su investigación "Propuesta de Aula Virtual como Estrategia de Aprendizaje para la Asignatura Dibujo Técnico. Unidad Educativa Mercedes Izaguirre de Corro" se planteó como objetivo proponer el uso de un aula virtual como aprendizaje para la asignatura dibujo técnico en estudiantes de segundo año de educación media, apoyándose de los distintos recursos tecnológicos.

El estudio fue presentado como proyecto factible, formulado sobre la base de una investigación de campo de carácter descriptivo; la población estuvo constituida por 30 estudiantes de tercer (3er) año y se aplicó un cuestionario de 11 ítems de alternativas hipotónicas de orden cerrado, siendo su respuesta si-no. Para la validez del instrumento se utilizó el juicio de expertos con la participación de cuatro profesores de la Mención. La confiabilidad se logró con la aplicación del método de cálculo interno de Kuder Richardson, donde se obtuvo un coeficiente de 0.61, esto indica una versión de ítems altamente confiable.

El análisis de los resultados se hizo de modo descriptivo, porcentual y de frecuencias; de ello se concluyó la necesidad de implementar la herramienta virtual Classroom para facilitar la productividad del aprendizaje de la asignatura Informática de tercer (3er) año de Educación Técnica, permitiendo así, servir de apoyo y guía para los estudiantes, con lo que se logró mejorar y enriquecer tanto la labor de los profesores como el rendimiento y la creatividad estudiantil, todo con el fin de mejorar la enseñanza en la institución.

Es importante resaltar estos antecedentes, ya que se hace el uso de los recursos tecnológicos mediante las estrategias virtuales, donde su principal objetivo es mejorar el rendimiento de los estudiantes y la labor del docente con la finalidad de servir de guía para el proceso educativo.

También conviene advertir que el estudio estuvo respaldado por la Teoría del Constructivismo de Piaget (1973). Dicho fundamento sostiene que en la educación los conocimientos se generan no sólo de los objetos mismos sino de las acciones ejercidas por el sujeto sobre éstos, así como de las propiedades que dichas acciones agregan a los objetos. La experiencia no es vista como una copia perceptiva del objeto, sino como el resultado de la interacción entre el sujeto y el objeto, es el factor determinante en el desarrollo de las estructuras cognitivas del sujeto.

Cabe considerar, ante lo expuesto, que el individuo construye el conocimiento partiendo de sus acciones del día a día, utilizando objetos que mediante la interacción con el sujeto desarrolle sus conocimientos a través del manejo de las herramientas tecnológicas que facilitan el proceso de enseñanza-aprendizaje.

Por otra parte, la investigación se basó en la Teoría de la Cibernética. Esta es una ciencia, nacida hacia 1948 e impulsada inicialmente por Norbert Wiener, que tiene como objeto "el control y comunicación en el animal y en la máquina" (s.n) o "desarrollar un lenguaje y técnicas que nos permitirán abordar el 
problema del control y la comunicación en general" (s.n). En 1950, Ben Laposky, un matemático de Iowa, creó los oscilones o abstracciones electrónicas por medio de un ordenador analógico: se considera esta posibilidad de manipular ondas y de registrarlas electrónicamente como el despertar de lo que habría de ser denominado computer graphics y, luego, computer art e infoarte. La cibernética dio gran impulso a la teoría de la información a mediados de los 60, la computadora digital sustituyó la analógica en la elaboración de imágenes electrónicas.

Cabe destacar que a mediados de los años 60 , el hombre ha venido ideando máquinas analógicas con ciertas capacidades en el campo tecnológico educativo, es decir con habilidades para el control y solución de problemas, así, influenciando los modelos cibernéticos que lo condujo a considerar un nuevo elemento en el modelo comunicativo matemático: la realimentación o "feed-back". Facilitando de esta forma una buena comunicación y desarrollo de cualquier actividad.

\section{Las Tecnologías de Información y Comunicación}

El fundamento de toda investigación consiste en el desarrollo de los elementos conceptuales más relevantes para sustentarla y definir puntos clave que promueven algunos autores precisando lo más relevante de los objetivos y metas que se pretenden alcanzar. En tal sentido las bases conceptuales se exponen las bases conceptuales que sustentan el estudio.

Las Tecnologías de Información y Comunicación (TIC) están ampliamente extendidas, ocasionando transformaciones en todos los ámbitos de la sociedad. En el campo educativo específicamente, las ventajas de proporcionar ambientes inteligentes de enseñanza-aprendizaje se hacen sentir en la necesidad de establecer un modelo educativo en la sociedad informacional que considere el auto aprendizaje, mediante entornos facilitadores de aprendizajes cognitivos, tal como el aprendizaje de oficio propuesto por Collins. Por otra parte, la necesidad de tomar en cuenta la diversidad desde todo punto de vista, en particular desde las Necesidades Educativas Especiales, para lograr el acceso incondicional a una educación en la sociedad de la información, conlleva a la puesta en marcha de iniciativas relacionadas al desarrollo de proyectos y recursos tecnológicos que puedan dar solución a esta situación.

En este sentido, las Tecnologías de la Información y Comunicación (TIC) juegan un papel preponderante, entendiéndose éstas según Cabero (1998), como las que giran en torno a tres medios básicos: la informática, la microelectrónica y las telecomunicaciones; pero giran, no solo de forma aislada, sino lo que es más significativo, de manera interactiva e interconectadas, lo que permite nuevas realidades comunicativas (p.198). Para Márquez (2000), las TIC son un conjunto de avances tecnológicos, posibilitados por la informática, las telecomunicaciones y las tecnologías audiovisuales, todas éstas proporcionan herramientas para el tratamiento y la difusión de la información y contar con diversos canales de comunicación. De todo lo anterior queda claro que las TIC son herramientas de gran utilidad que facilitan el acceso, manejo, procesamiento y difusión de la información en una sociedad que cada día está más interconectada y que exige de sus miembros nuevas actitudes y aptitudes frente al conocimiento.

Ante los argumentos planteados es de hacer notar las características de las TIC, las 
cuales son la inmaterialidad, interactividad, instantaneidad e innovación; pues es de carácter innovador y creativo, ya que dan acceso a nuevas formas de comunicación; además, tienen amplio dominio y beneficio, en mayor proporción al área educativa, ya que la hace más accesible y dinámica; se relacionan con mayor frecuencia con el uso de la Internet y la informática. En resumidas cuentas, la herramienta TIC, es de importancia en la educación, ya que le proporciona fuentes de información a los docentes-estudiantes en todo proceso de enseñanza-aprendizaje de manera interactiva efectiva.

\section{MÉTODO}

Esta investigación se enmarcó en el carácter descriptivo con apoyo de investigación de campo, ya que buscó comprender el registro e interpretación directa de los fenómenos, utilizando los hechos necesarios para así encontrar sus soluciones.

El diseño pretende alcanzar respuestas a las interrogantes presentadas, pues permite desglosar las estrategias que se adoptan para generar información exacta e interpretable. Tal como lo menciona Arias (2006), el diseño de la investigación consiste en "la estrategia general que adopta el investigador para responder al problema planteado" (p. 32).

Con relación al tipo de diseño de la investigación, se ubica dentro del estudio de campo, debido a que no se manipulan las variables, simplemente se observó el fenómeno tal cual como ocurren en su contexto natural, recolectando datos directamente de la realidad donde se manifiesta el problema de esta institución académica, lo cual nos arrojó la necesidad de recabar información sobre la implementación de Classroom como herramienta que ofrece la plataforma Google App, teniendo en consideración que las TIC se pueden utilizar para complementar el proceso de enseñanza-aprendizaje de los estudiantes.

Arias (2006) explica que la investigación de campo se refiere a "la recolección de todos directamente de los sujetos investigados, o de la realidad donde ocurren los hechos, sin manipular o controlar variables alguna; es decir, el investigador obtiene la información, pero no altera las condiciones existentes" (p. 55).

Por otro lado, es pertinente acotar un elemento constitutivo metodológico: La población. Ésta es el conjunto finito o infinito de elementos con características comunes, para los cuales serán extensivas las conclusiones de la investigación. Esta queda limitada por el problema y los objetivos del estudio.

En este caso, dado el tipo de investigación, la población estuvo constituida por treinta (30) estudiantes del tercer "3er" año, sección "A" de la E.T.R "Monseñor Gregorio Adam", Naguanagua, estado Carabobo. Se entiende por muestra al subconjunto representativo y finito que se extrae de la población accesible. Representa una pequeña población, es decir, una parte de la población objeto de estudio, de allí es importante asegurarse que los elementos de la muestra sean lo suficientemente representativos de la población que permita hacer generalizaciones. Por su parte Hernández, Fernández y Baptista (2014) expresan que "si la población es menor a cincuenta (50) individuos, la población es igual a la muestra" (p. 69). Es decir, la muestra es finita y quedó conformada por treinta (30) estudiantes.

Con relación al presente trabajo se seleccionó la encuesta como la técnica de recogida de datos, la cual es definida por Hurtado (2010), como "una técnica de recolección de datos, en investigaciones de 
campo, que consiste en obtener información de una muestra representativa de una población" (p. 76). Por otra parte, se seleccionó el cuestionario como instrumento de recolección de datos, según Arias (2006), "se realiza de forma escrita mediante un instrumento o formato en papel contentivo de una serie de preguntas contestada en negaciones y afirmaciones" (p. 56).

Se empleó un cuestionario de respuestas dicotómicas, donde la validez y confiabilidad se determinó por el coeficiente Kuder Richardson, el mismo estuvo compuesto por once (ll) ítems, con opción de respuestas alternativas de sí y no; lo cual indica que el instrumento fue dicotómico.

La validez y la confiabilidad son constructores de una investigación desde aspecto positivista para otorgarle a los instrumentos y a la información obtenida, exactitud y firmeza necesarias para efectuar las generalizaciones de los aciertos derivados del análisis de las variables en estudio. Por tanto, para Ruíz. (2002)

La validez representa la posibilidad de que un método de investigación sea capaz de responder a las interrogantes formuladas. La confiabilidad designa la capacidad de obtener los mismos resultados de diferentes situaciones.

Que significa:

Kr: coeficiente de la confiabilidad

n: número de ítems del instrumento

Vt: varianza total del instrumento

Pq: sumatoria de la varianza individual

Del ítem del instrumento
La confiabilidad no se refiere directamente a los datos, sino a las técnicas de instrumentos de medida y observación, es decir, al grado en que las respuestas son independientes de las circunstancias accidentales de la investigación. (p. 134)

La validez de la presente investigación se realizó por parte de (2) docentes del departamento en donde se verificaron los ítems del instrumento y si estos fueron representativos. Al respecto, Balestrini (2006) plantea:

Una vez que se ha definido y diseñado los instrumentos y procedimientos de recolección de datos, atendiendo al tipo de estudio de que se trate, antes de aplicarlos de manera definitiva en la muestra seleccionada, es conveniente someterlos a prueba, con el propósito de establecer la validez de estos, en relación al problema investigado. (p.140)

Para la medición de los ítems presentados se aplicó el coeficiente de Kuder y Richardson (KR20), ya que permite obtener la confiabilidad a partir de los datos conseguidos.

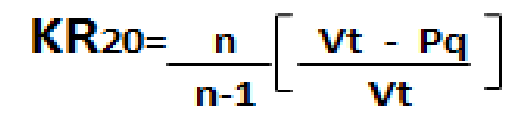

$\mathrm{KR}_{20}=0,61$ 
Tabla 1. Criterios de decisión para la confiabilidad de un instrumento

\begin{tabular}{llllll}
\hline \multirow{3}{*}{ Rango } & \multicolumn{5}{c}{ Rango de confiabilidad (Kuder-Richardson 20) } \\
\cline { 2 - 6 } & $0-0,20$ & $0,21-0,40$ & $0,41-0,60$ & $0,61-0,80$ & $0,81-1$ \\
& Muy baja & Baja & Media & Alta & Muy Alta \\
\hline
\end{tabular}

Fuente: Ruíz (2002)

Como conclusión se obtuvo una confiabilidad de 0,61 para el coeficiente de Kuder y Richardson (KR), lo que quiere decir que se consiguió una confiabilidad "Alta".

\section{RESULTADOS}

Lo que se pretendió con la investigación fue dar a conocer las ventajas y beneficios que ofrece la Tecnología como estrategia virtual en el ámbito educativo. Por lo tanto, el instrumento aplicado a los estudiantes de 3er año de E.T.R "Monseñor Gregorio Adam", arrojó que sí es necesario el uso de esta plataforma en la asignatura de Informática, ya que la Institución antes mencionada cuenta con los recursos precisos para realizar cada clases con el apoyo del Proyecto Canaima, permitiéndole así una buena interacción y comunicación entre docente-estudiante.

Además estas herramientas le permiten al estudiante ser organizado a la hora de realizar cada actividad propuesta por el docente para ser más productivo y eficaz a la hora de dar el proceso de enseñanza-aprendizaje. Con la aplicación de este instrumento se puede corroborar que esta plataforma sirve de apoyo al docente, no solo por el beneficio que le aporta al docente, sino porque desarrolla las destrezas de los estudiantes en la adquisición de nuevas habilidades y perfeccionamiento de sus competencias académicas. A su vez apoya la concepción del aprendizaje constructivista por cuanto está centrada en el estudiante como sujeto de su propio aprendizaje.

La propuesta de la plataforma Classroom como herramienta virtual en la asignatura de Informática brinda beneficios, tanto al docente como a los estudiantes, porque consiente mejorar el proceso de enseñanza-aprendizaje, rompiendo así con los paradigmas de la educación tradicional, permitiéndole al docente actualizarse y estar constantemente capacitado, a la hora de planificar una clase; sin embargo a los estudiantes les permitirá mejorar sus conocimientos previos, obteniendo un impacto positivo de un aprendizaje continuo y constructivista.

A continuación una síntesis explicada gráficamente: 
Barra de direcciones

\section{-. Google Classroom}

Utilice

l. Abra su correo Gmail.

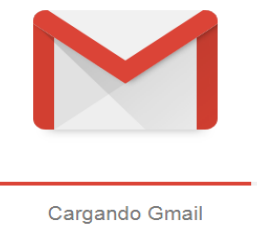

2. Aplicación

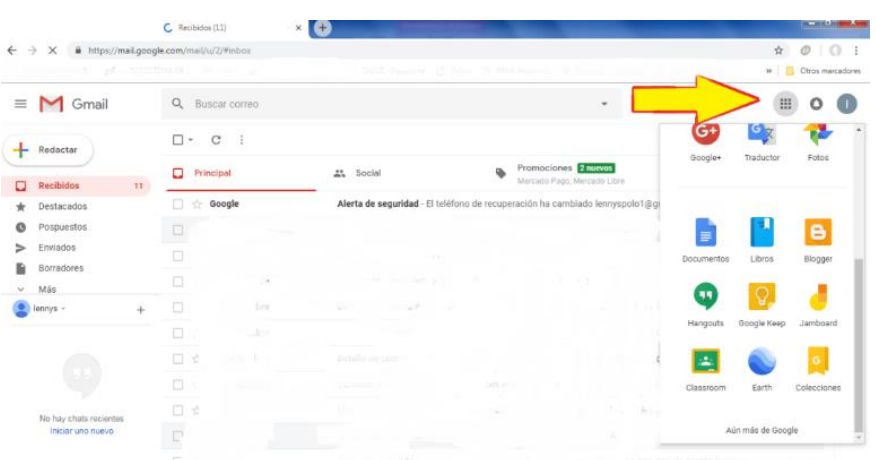

3. Inicio de la herramienta virtual Classroom.

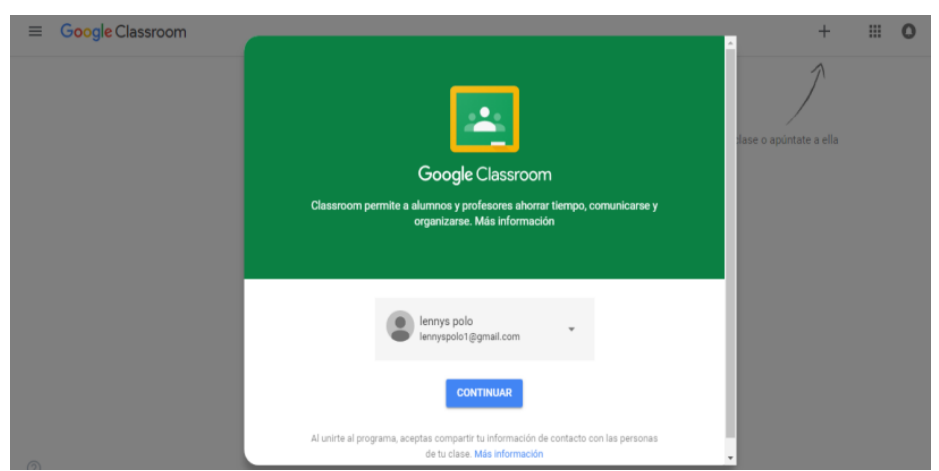

Acción

Obligatoriamente se debe tener una cuenta en Gmail.

La flecha indica todas las aplicaciones de Google Gmail, donde encontrará el logo de Classroom.

El docente aceptará los lineamientos de Classroom para crear su perfil. 


\begin{tabular}{cc}
\hline Utilice & Acción \\
\hline
\end{tabular}

\section{Como crear la clase}

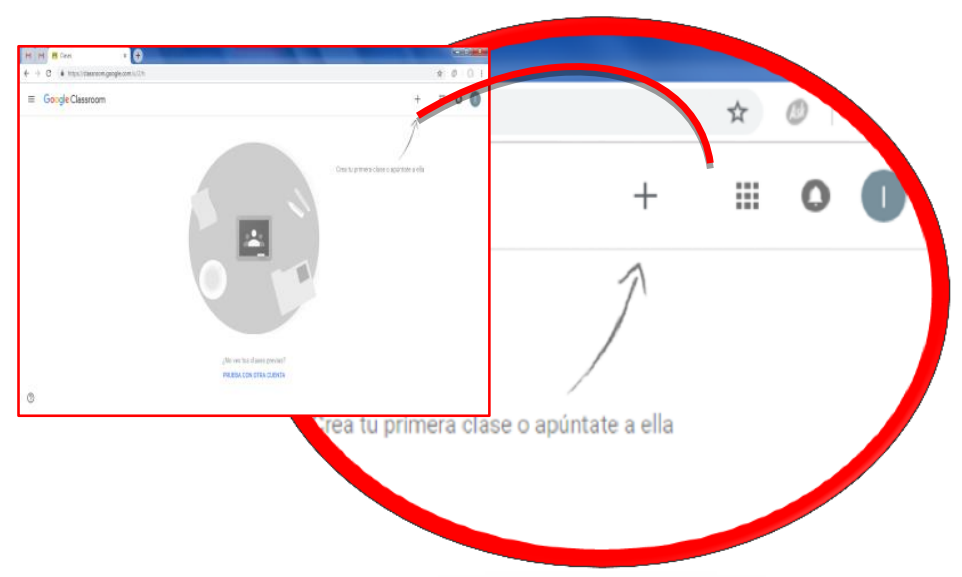

5. Ingresar la informacion correspondiente a la clase

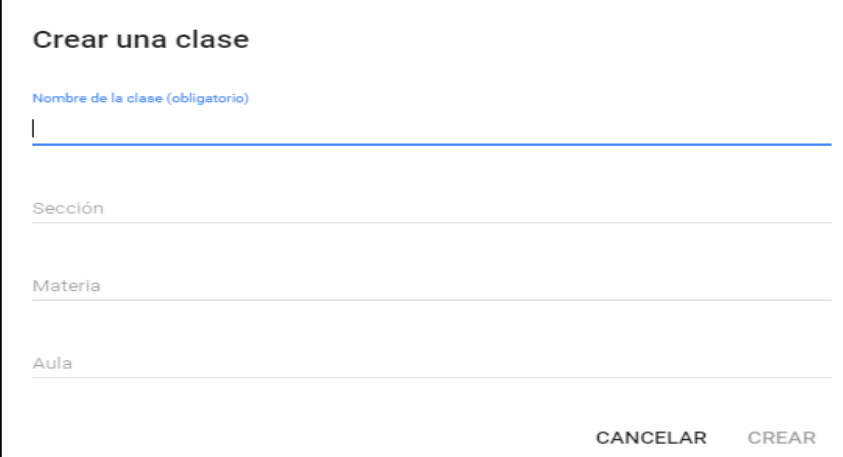

6. Clase creada

$$
\equiv \text { Google Classroom }
$$

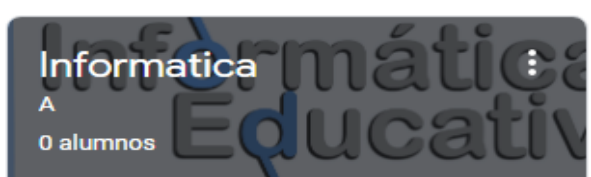

El docente debe hacer click al signo positivo que se ve en la imagen para crear su clase virtual.

El docente ingresará la información correspondiente a la clase.
La clase se creará y aparecerá en la página de inicio junto con las otras clases. 


\section{Utilice}

7. Envío de código a los estudiantes

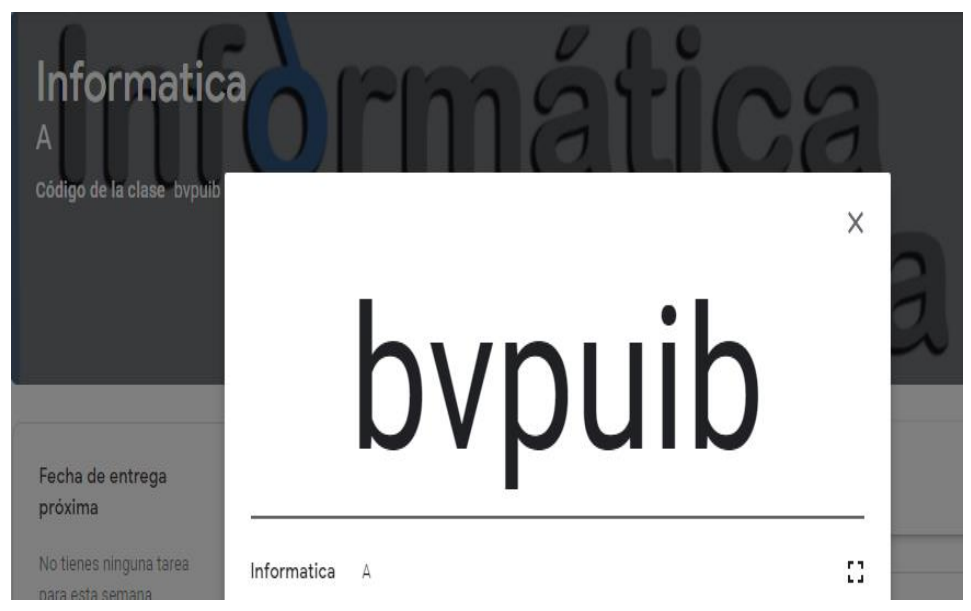

Acción

El docente deberá enviar un código que le arroja la apertura de la clase para poder ingresar a los estudiantes al salón de clase virtual.

Figura l. Infografía.

\section{CONCLUSIONES}

La educación va de la mano con la tecnología porque, en conjunto, se convierte en un modelo educativo innovador. Adicionalmente, permite a los estudiantes una educación eficaz utilizando los recursos didácticos necesarios para fortalecer la formación y conocimiento tanto del docente como del estudiantado.

Por lo tanto se propuso el uso de esta herramienta virtual para hacer del proceso de enseñanza-aprendizaje factible, en donde los estudiantes individualmente puedan subir y realizar sus actividades para ser evaluados por el docente, teniendo en cuenta que no se hace ningún gasto ni uso de papel. Además se pudo evidenciar que la institución cuenta con un laboratorio de informática, el cual consta con 20 computadoras del proyecto Canaima, en ellas se puede iniciar la propuesta de usar Classroom como una herramienta virtual significativa para el proceso de enseñanzaaprendizaje. Dentro de este marco de ideas esta plataforma cuenta con los siguientes beneficios: a) Facilidad para crear; b) ahorra tiempo; c) mejora la organización; d) facilita la comunicación, gratuita y segura.

La propuesta presentó como objetivo general: Ofrecer una plataforma Classroom como herramienta virtual en la asignatura de Informática dirigido a los estudiantes del 3er año "A" de la E.T.R "Monseñor Gregorio Adam”, ubicado en el Municipio Naguanagua del estado Carabobo. Asimismo los específicos fueron: Brindar a los estudiantes del 3er año "A" de la E.T.R "Monseñor Gregorio Adam" esta herramienta que le permita realizar las actividades de manera factible, facilitar a los estudiantes estos recursos tecnológicos como estrategias para el desarrollo de las asignaciones establecidas por el docente en el E.T.R "Monseñor Gregorio Adam” y fomentar en los estudiantes el uso de las TIC como recursos para el fortalecimiento de su conocimiento y formación en la institución referida. 
Posterior a ello, como es sabido todo proyecto factible debe tener una misión y visión de la propuesta, por lo cual se plantea como misión: Facilitar la realización de las actividades propuesta por el docente a los estudiantes, utilizando las herramientas virtuales, como recurso didáctico en el proceso educativo. Además de la visión: Mejorar el aprendizaje de los estudiantes partiendo del conocimiento previo, en conjunto con las TIC para lograr un aprendizaje significativo, positivista y constructivista.

Precisando de una vez, la siguiente propuesta se basó en la importancia de la tecnología, información y comunicación como estrategia de un aula virtual, como recurso didáctico en la asignatura de informática, ya que por medio de ella se puede impartir clases a distancia y evaluar mediante una plataforma, obteniendo como objetivo principal, proponer Classroom como herramienta virtual dirigida a los estudiantes para lograr un aprendizaje significativo. Este recurso tiene, dentro de una de sus funciones, el intercambio de conocimientos entre docente y estudiantes como un eje factible en las actividades no presenciales, controlado por el docente con base la comunicación.

Asimismo, la juventud venezolana hoy en día, al igual que la sociedad globalizada, gira alrededor de la tecnología. Particularmente en la República Bolivariana de Venezuela, a través de Ministerio del Poder Popular para la Educación, hay un proyecto en ejecución llamado "Canaima", con el objetivo de apoyar la formación integral con dotación de computadoras portátiles escolar con contenidos educativos para los docentes y estudiantes del subsistema de educación primaria, media y técnica; extendiéndose a los demás sistemas educativos, conformado por instituciones públicas nacionales, estadales, municipales, autónomas y las privadas subsidiadas por el Estado.

Análogamente, los recursos didácticos digitales tienen una peculiaridad que no existe en los recursos educativos tradicionales; no es igual leer un documento impreso, cuyo discurso fluye en forma lineal, que leer el mismo en forma digital, escrito en formato hipertextual, estructurado como una red de conexiones de bloques de información, donde el lector puede elegir su lectura de forma personalizada, utilizando la herramienta de interés.

De tal manera que los materiales digitales se denominan recursos educativos digitales apuntando al logro de un objetivo de aprendizaje, como lo planteado en la presente propuesta al ofrecer una herramienta virtual como recurso didáctico de la asignatura de Informática, dirigida a los estudiantes del 3er año de la E.T.R Monseñor Gregorio Adam.

Dadas las condiciones que anteceden, se determinó el estudio de la propuesta, partiendo de la factibilidad técnica, la cual está relacionada con todos aquellos aspectos necesarios que permitan la realización de las actividades o procesos que requiere esta propuesta factible. En tal sentido al evaluar el proyecto resultó factible, donde se cuenta con la infraestructura tecnológica y con las políticas académicas de diseño instruccional para llevar a cabo la implementación de una herramienta virtual como recurso didáctico en la asignatura de Informática. Asimismo se refiere a los recursos económicos y financieros necesarios para desarrollar o ejecutar las actividades o procesos para obtener los recursos básicos que deben considerarse con el costo para su debida implementación, en tal sentido, se realizaron los estudios respectivos y la investigación se consideró factible económicamente. 
Es importante resaltar que los educandos que conforman el universo de estudio de este caso, no pueden permanecer aislados a las exigencias actuales de tiempo y lugar, propio de una sociedad globalizada y competitiva, de las que por su propia naturaleza resulta imposible deslastrarse la juventud contemporánea, por constituir parte de su matriz de origen, en virtud de lo cual es imprescindible dotarles de herramientas cónsonas con el proceso de enseñanza-aprendizaje.

Como educadores en ejercicio cónsonos con la responsabilidad profesional, es necesario agotar recursos para no solo dotar a los estudiantes de las herramientas requeridas para la comprensión de los objetivos generales y específicos que conforman las unidades programáticas del pensum de estudios respectivos, sino además para lograr el desarrollo eficaz del proceso cognitivo requerido para el cumplimiento exitoso de la meta propuesta: "educar para aprender", de ahí el porqué de la presente investigación.

Las exigencias de la sociedad actual obligan a la comunidad educativa a actualizarse para el efectivo logro de sus fines. Es por ello que se considera más que oportuno el uso de la plataforma Classroom como instrumento para consolidar el logro del proceso enseñanza- aprendizaje en este ámbito, de manera efectiva, oportuna y eficaz.

Por otra parte, es relevante el dominio del idioma inglés, puesto constituye hoy día una necesidad y exigencia importante, más allá de cualquier consideración política e ideológica, en razón a que como idioma su uso e implementación se ha universalizado para referirse al empleo de tecnología de punta y conocimientos de importancia económico y tecnológico de orden mundial, sobre todo en el micro universo de la informática, que requieren, incluso desde el ámbito de la cultura general, ser conocidos e implementados, razón por la cual se necesita hoy día de la mayor precisión posible para incorporarla al proceso enseñanzaaprendizaje, logrando con ello colaborar a la construcción del ciudadano del futuro, de ahí precisamente la necesidad de ser tomada en cuenta la implementación de la plataforma Classroom.

\section{REFERENCIAS}

Agudo, A. (2016). Aula Virtual como Estrategia de Aprendizaje para la Asignatura Dibujo Técnico "U.E Mercedes Izaguirre de Corro". Recuperado: http://mriuc.bc.uc.edu.ve/handle/123456 $789 / 3121$

Arias, F. (2006). El Proyecto de Investigación. Introducción a la metodología científica. Venezuela: Editorial Episteme

Balestrini, M. (2006). Cómo se elabora el Proyecto de Investigación. Venezuela: Consultores Asociados

Cabero, J. (1998): Impacto de las nuevas tecnologías de la información y la comunicación en las organizaciones educativas, en Lorenzo, M. y otros (coords): Enfoques en la organización y dirección de instituciones educativas formales y no formales, Granada, Grupo Editorial Universitario, 197-206

Constitución de la República Bolivariana de Venezuela. Caracas. Gaceta Oficial $\mathrm{N}^{\circ}$ 5.453 del 24 de Marzo de 2000

Hernández, R., Fernández, C. y Baptista, P. (2014). Metodología de la Investigación. México: Editorial McGraw-Hill

Hurtado, J. (2010). Metodología de la Investigación Holística. Venezuela: Servicios y Proyecciones para América Latina

Ley Orgánica de Ciencia, Tecnología e Innovación. Gaceta Oficial № 38.242 del 03 de Agosto de 2005 
Márquez, G. (2000). Grandes aportaciones de las TICs" (23/03/2008) ,0l:32. Recuperado: http://www.pangea.org/peremarquez/tic .htm

Piaget, J. (1973) La representación del Mundo en el Niño. Madrid: Morata.

Ruíz, C. (2002). Instrumentos de Investigación Educativa. Procedimientos para su Diseño y Validación. Venezuela: CIDEG

Sánchez, C. (2017) Flipped Classroom: la clase invertida una realidad en la
Facultad de Ciencias de la Educación en la Universidad de Málaga. Disponible en https://www.uma.es/innoeduca/info/104 324/tesis-finalizadas/

Unesco (2008). Estándares de Competencia en TIC para Docentes. Recuperado: http://portal.unesco.org/es/ev.php.URL I $\mathrm{D}=41553 \&$ URL_DO=DO_TOPIC \& URL_SECTION=201.html

Wiener, N. (1948). Teoría de la Cibernética. Recuperado:

http://www.google.com.co/imgres?imgur l=http://www.rolfbehncke.cl/I 